\title{
Etude des possibilités et des conditions nécessaires pour la création de réservoirs artificiels en Sardaigne par rapport aux caractéristiques physiques de l'lle ${ }^{(1)}$
}

Dans cette époque de Renaissance technique chez nos voisins, l'hydrotechnique semble être la branche qui les intéresse le plus et qui effectivement fait de très sérieux progrès. Par centaines se comptent les ouvrages d'art disséminés dans le pays tout entier. On compte par milliards les mètres d'eau retenus et asservis par la volonté et la valeur des techniciens, qui veulent e. réussissent à doter leur pays de l'énergie qui lui est nécess?ire pour le développement de son industrie tout en l'émancipant at le rendant indépendant de l'étranger au point de vue combustible employé pour la production de la force motrice.

Ce problème étant vital, toutes les mesures sont prises pour que les services responsables soient aussi bien organisés que possible,

On retrouve en effet dans chaque branche de l'activité hydrolechnique une ample documentation sur les conditions générales et hydrologiques des diverses régions ainsi que sur les endroits qui sont à priori destinés à former des réservoirs artificrels.

Nême lorsque les probabilités de réalisation sont minimes les ctudes préliminaires sont scrupuleusement exécutés. Cette documentation complète mise à la disposition du Conseil Supérieur des Travaux Publics permet à celui-ci de désigner sans hésitation les travaux qu'il conviendrait d'entreprendre au as où une région bien déterminée en aurait besoin ou des allocations dans le but d'offrir en échange d'un travail, si on a un excès de main d'œuvre à employer, ou bien au cas où l'on aurait du capital à placer. Ces bureaux d'étude et de documentation s'étendent sur tout le pays, divisés en groupe suivant les régions

$$
\text { *** }
$$

Les conditions climatéorologiques de la Sardaigne sont particulièrement défavorables au développement de l'agriculture et à la production de la force motrice. En cette période où les difficultés financières sont grandes partout, les régions passives s'en ressentent encore plus et les conditions de vie y sont beaucoup plus pénibles. Cette partie de l'Italie ne peut être ainsi laissée à elle-même dans les difficultés que la nature lui impose, car les habitants de la Péninsule ne peuvent oublier le rôle qu'ont joué l'Ile et ses habitants dans l'histoire italienne ainsi que la magnifique fidélité des Sardaignois dans la dernière guerre ct les dirigeants responsables de l'avenir de l'Italie en tiennent bien compte. D'autre part cette grande lle est un point stratégique de la plus haute valeur dans la mer Méditerranéenne, ce que l'on n'oublie pas non plus comme nous le verrons par la suite.

La revalorisation de l'agriculture en Sardaigne est le principal problème qui peut intéresser ses dirigeants, mais qui n'est pas facile à répondre : il suffit pour cela de savoir que les précipilations atmosphériques y sont trés irrégulières, (plus de la moitié des précipitations annuelles se placent dans les deux mois: avril et octobre), et que, une fois sur quatre, se présente l'éventualité d'un mois de mai sans une goutte de pluie, cela entraîne comme conséquence fatale la perte totale de la récolte du grain. Les autres récoltes ne sont pas plus épargnées. On conçoit combien grand doit être l'attachement des habitants de cette terre antique pour continuer avec tenacité à lutter contre toutes ces adversités ; la configuration orographique n'est pas plus favorable.

Le relief est très montagneux et rocheux avec des montagnes dont les flancs très raides se précipitent directement dans la mer. Les cours d'eau sont peu importants et pour la plupart secs en été, Dans ces conditions la distribution de l'eau dans l'lle est nettement insuffisante pour résoudre le problème de la transformation des cultures, il ne reste done pour fournir cette eau qui y est nécessaire, qu'à créer dans l'lle des réservoirs sous forme de lacs artificiels, pour lesquels, fort heureusement la nature du terrain se prête particulièrement bien. Des ouvrages magnifiques ont été déjà réalisés, dont le plus bel exemple est bien celui sur le Tirso, qui forme le plus grand lac artificiel en Europe. Ajoutons encore que la nature de ces terrains a le grand avantage de ne pas mettre en danger l'existence des réservoirs, comme c'est le cas dans d'autres régions du Royaume, où leur existence peut ètre mise en jeu à brève échéance. II suffit de signaler le cas où à la suite d'une étude récente, de relevés trés soigneusement effectués on a pu évaluer, dans un réservoir des Apennins de construction récente, à $1500 \mathrm{~m}^{2}$ par $\mathrm{km}^{2}$ et par an l'apport de matière solide, de sorte que pour ce réservoir qui fut créé pour la production de l'énergie et comme perspective future l'utilisation pour l'irrigation, tout espoir d'un fonctionnement durable dans ce sens doit ètre complètement abondonné.

Le programme élaboré pour la Sardaigne en fait de construction des barrages prévoit l'accumulation de l'eau dans le rapport de $40 \%$ de celle qui se précipite sur l'lle et par les différents cours d'eau qui actuellement s'écoule inutilisés à la mer, ce qui représenterait au total une retenue de 2 milliards de mètres cubes. Ces barrages devraient être en général du type à gravité, en atteignant quelquefois la hauteur de $50 \mathrm{~m}$. même si cette solution n'est pas toujours la plus économique. On insiste sur la construction éventuelle de barrages à gravité et cela à cause de la moindre vulnérabilité de ces derniers, au cas d'une offensive aérienne en cas de guerre. On ne peut nier que les études soient complètes en tout point de vue. Cette considération de stratégie dans l'lle préoccupe beaucoup le Conseil Supérieur des Travaux Publics, puisque dans les calculs les possibilités de production d'énergie hydroélectrique qui puissent satisfaire les besoins de l'Ile, si elle venait a être abandonner à elle-même constitue un facteur essentiel dans l'ilaboration des avants-projets.

Les centrales thermiques inutilisables en cas de guerre à cause du manque très probable de combustible seront remplacées par des centrales hydroélectriques, construites dans des cavernes ou des sous-sols et, qui de ce fait, seraient soustraites aux attaques aériennes. Un magnifique exemple de construction de ce genre exécuté et mis en service nous est donné par la centrale de Coghinas à Muzzone. L'lle pourrait ainsi être complètement abandonnée à elle-même qu'elle pourrait se suffire quand à la production de l'énergie qui lui est nécessaire.

(1) D'après l'étude parue dans Annali dei Lavori Publici-Mai 1934 
Toutefois puisque le but d'irrigation prévaut sur celui de la production de l'énergie, celle-ci devra être subordonnée à celle là.

LE POSSIBILITA PER LA CREAZIONE'DI SERBATOI ARTIFICIALI IN SARDEGNA IN RAPPORTO ALLE CARATTERISTICHE FISICHE DELL ISOLA

SCHEMA DELLIDROCRAFIA SUPERFCIALE DELLA SARDEGNA CON INDICAZIONE DEI SERBATOI ARTIFICIALI FUNZIONANTI E POSSIBIL

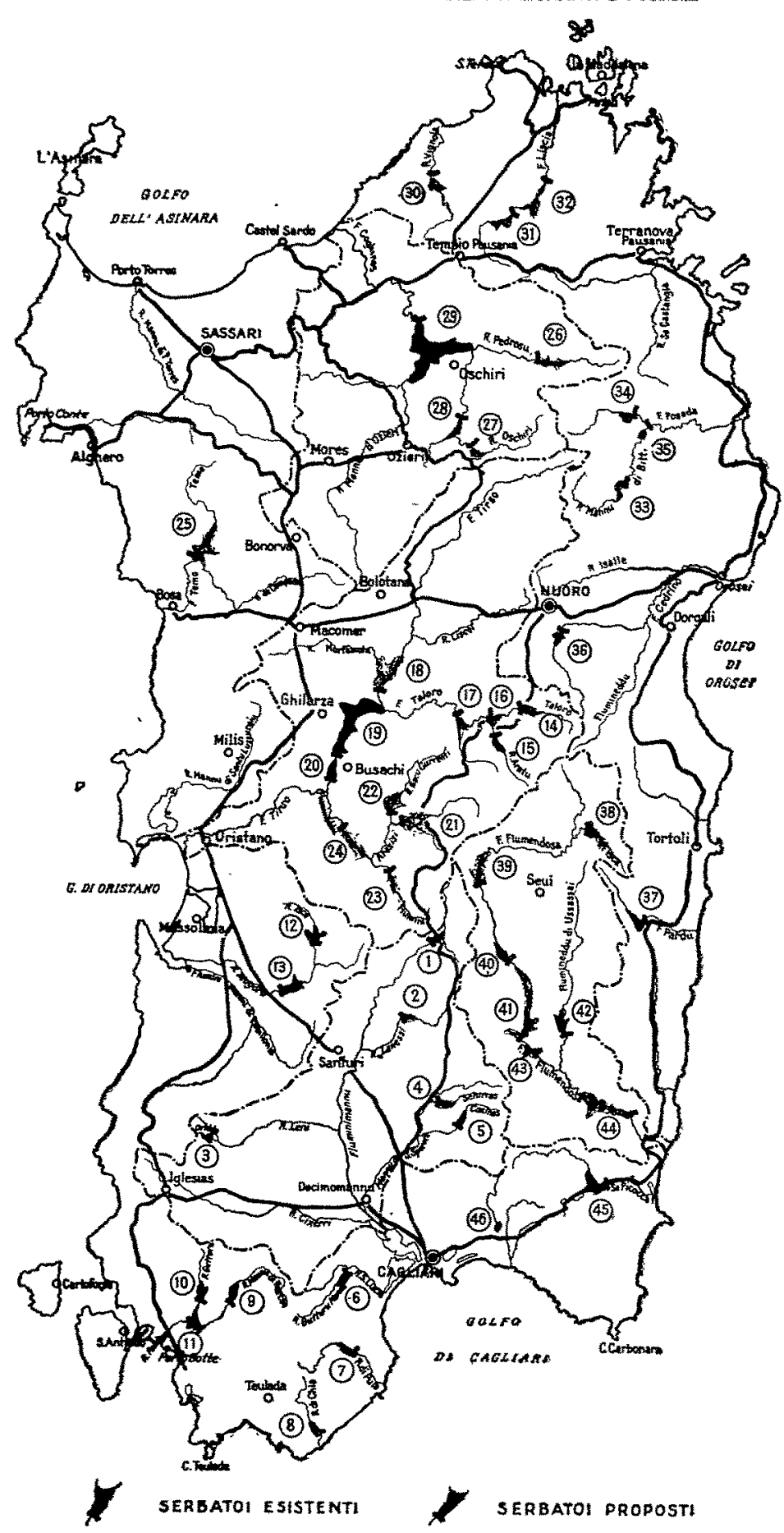

La possibilita per la creazione di serbatoi artificiali in Sardegna in rapparto alle caratteristiche fisiche ell' isola.

Schema dell' idrografia superficiale della Sardegna con mndicazione dei serbatoi artificiali funzionanti e possibili. de réservoirs artificiels en Sardai gne, en rapport des caractéristiques physiques de l'île.

Carte hydrographique de la Sardaigne avec indication des réservoirs artificiels en service et en projet.

Réservoirs existants

Reservoirs proposés
Pour la revalorisation agraire de l'Ile on a évaluć qu'il faut par hectare de terrain irrigué une réserve de 10 milles mètres cubes d'eau par an, quantité qui correspond a la distribution d'eau nécessaire aux différentes cultures où prévallent généralement les fourrages, qui, de l'avis des personnes compétentes, représentent la pierre angulaire de la revalorisation agraire de l'Ile.

La surface ainsi révalorisable serait de 150.000 hectares.

$$
*^{*} *
$$

Le service hydrographique de l'lle se divise en huit zones, pour chacunes d'elles nous exposerons très brièvement la situation actuelle, ainsi que les possibilités d'une organisation future.

1) Bassin du Flumini Mannu : - C'est dans cette zone que se trouve la plus grande partie du terrain irrigable en Sardaigne et sa transformation représenterait une véritable révolution économique pour l'He. Mais malheureusement plus de la demi-longueur de ce cours d'eau s'effectue en plaine, et près de son embouchure se trouvent plusieurs marais. Il n'y a done guère la possibilité de créer dans ce bassin des réservoirs importants, sauf éventuellement un qui serait relié au barrage de l'Alto Flumendosa à Is Barrocus dont la capacité pourrait atteindre 14 millions de $\mathrm{m}^{3}$ si la hauteur du barrage atteint $50 \mathrm{~m}$.

Plus loin il sera question du moyen d'obvicr cet inconvénient à défaut des ressources locales.

2) Bassin entre le Flumini Manna el le Tirso: - Cette zone peut être divisée en trois parties à caractéristiques différentes :

a) Cette première partie comprend tous les cours d'eau débouchants sur le littoral oriental et méridional du Sulcis et elle est constituée pour la plupart par des terrains imperméables. Le plus important des torrents qui $s^{\prime} y$ trouvent est le Rio di Palmas avec ses $476 \mathrm{~km}^{2}$ de bassin. On y prévoit trois réservoirs : le premier sur le Rio Manmu di Narcas à Serra Murdegu, dont la capacité atteindrait $16,5 \times 10^{6} \mathrm{~m}^{3}$; le second s'obtiendrait au moyen d'un barrage sur le Rio Gutturu de Ponti dont la capacité serait de $21,15 \times 10^{6} \mathrm{~m}^{3}$; le troisième barrage est prévu à la gorge de Monti Pranu sur le Rio di Palmas lui-même, la capcccité du réservoir ainsi formé serait de $24 \times 10^{6} \mathrm{~m}^{3}$.

Ces ouvrages permettraient l'irrigation et l'exploitation rationnelle de 8.000 hectares, dans une zone ou existaient déjà des agglomérations rurales ce qui activeraient sensiblement la prospérité dans la région.

On prévoit aussi dans les autres bassins de cette zone trois autres réservoirs artificicls d'eau savoir: un de $9,2 \times 10^{6} \mathrm{~m}^{3}$ sur le Rio Gutturu Mamu à Lurdagnu ; un autre de $15 \times 10^{6} \mathrm{~m}^{3}$ sur le Rio di Pula à Crippeddu Marrone et le troisième, aussi de $15 \times 10^{6} \mathrm{~m}^{3}$, sur le Rio di Chiu à Predi Bonu. Dans l'ensemble ils permettraient l'irrigation d'une étendue totale de 5.000 hectares.

b) La deuxième partie, qui constitue la principale région minière de l'Ile, a des cours d'eau d'importance très limitée et soit à cause de sa situation topographique, soit à cause de la structure géologique elle ne se prête pas 
à la formation de réservoirs artificiels d'une importance quelconque.

c) La troisième partie est essentiellement constituée par les bassins du Flumini Mannu di Pabillonis et du Rio di Mogoro. Ce n'est que dans ce dernier qu'il est possible de constituer des réservoirs artificiels. En effet on y prévoit la construction d'un barrage qui formera un réservoir de $8 \times 10^{6} \mathrm{~m}^{3}$ destiné à la production de l'énergie nécessaire à cette région. Sur ce Rio di Mogoro, a été récemment exécuté à St-Vittoria un, réservoir dont la capacité est de $22.5 \times 10^{6} \mathrm{~m}^{3}$ qui est prévu comme régulateur de débit pendant les fortes crues, il n'est toutefois pas dit qu'à l'avenir il ne puisse servir à d'autres fonctions, si de nouvelles exigences se précisent pour l'agriculture. La construction de ce barrage, qui est solidement encastré dans une gorge constituée par des calcaires très compacts, est parfaitement réussie.

3) Bassin du Tirso : Le Tirso est le cours d'eau de Sardaigne qui a le bassin hydrographique le plus étendu $\left(3.376 \mathrm{~km}^{2}\right)$.

Pour la formation de réservoirs artificiels, les affluents de droite ainsi que la partie haute du cours d'eau ne présentent aucun intérèt à cause de la qualité du terrain, par contre, à ce point de vue, sont très importants : le cours moyen du Tirso ainsi que ceux du Taloro et du Flumineddu et cela d'autant plus que l'embouchure du Tirso s'effectue en plaine appelée Campidano Nord di Cagliari et qui constitue la plus vaste ressource agricole de la Sardaigne.

L'importance primordiale de cette situation pour l'Ile explique l'existence de barrages sur ce cours d'eau et en particulier ceux de St-Chiara d'Ula et de Busachi, la capacité du premier s'élève à $374 \times 10^{6}$, le plus important d'Europe et qui est l'orgueil de l'hydrotechnique italienne, le second n'a qu'une capacité de $10^{6} \mathrm{~m}^{3}$ et il n'est destiné qu'à la régulation journclière du précédent. Le débit disponible du réservoir de St-Chiara, fut d'abord évalué à $20 \mathrm{~m}^{3} \mathrm{sec}$; mais par la suite on a constaté que le chiffre exact est $15,48 \mathrm{~m}^{3} \mathrm{sec}$, ce qui est suffisant pour l'irrigation des 20.000 hectares prévus par les avant-projets ; mais insuffisant pour les 40.000 hectares que l'on aurait à irriguer, si on voulait étendre cette action bienfaisante à tout le Campidasso. On peut remédier à cette déficience, soit en réservant le débit total de St-Chiaro pour la période d'irrigation et en subvenant à la production de l'énergie pendant l'hiver au moyen d'autres installations, soit en effectuant une régulation ultérieure au moyen d'autres réservoirs aménagés sur le Taloro et sur le Flumineddu. Pour cela on a déjà étudié complètement la réalisation éventuelle des réservoirs artificiels suivants : celui de Casa Barabba dont la capacité utile est de $27,36 \times 10^{6} \mathrm{~m}^{3}$, celui de Pedras Fittas avec une capacité de $12 \times 10^{6} \mathrm{~m}^{3}$, celui de St-Pietro dont la capacité de $27 \times 10^{8} \mathrm{~m}^{3}$, celui de Cucchina di Org a d'une capacité de $30 \times 10^{6} \mathrm{~m}^{3}$, et finalement le plus important celui du Monte Mundregu dont la capacité serait de $138 \times 10^{6} \mathrm{~m}^{3}$, les différents barrages, destinés à former ces réservoirs auraient des hauteurs qui varieraient entre 36 et $59 \mathrm{~m}$

4) Ce quatrième bassin est compris entre celui du Tirso et celui du Coghinas. Cette région est relativement assez riche 'n sources, qui ont été utilisées pour l'irrigation locale, mais leur débit n'est pas suffisant pour la région. Dans ce bassin le principal cours d'eau est le Temo sur lequel est prévu la construction d'un réservoir obtenu au moyen d'un barrage de $44 \mathrm{~m}$. de hauteur, situé à Ponte Enas et dont la capacité serait de $78 \times 10^{8} \mathrm{~m}^{3}$. Toutefois cette réserve ne serait d'aucune utilité pour l'agriculture, étant donné qu'il n'y a pas aux environs immédiats des terrains cultivables ; mais en revanche on pourrait obtenir un important centre de production d'énergie. On prévoit en effet qu'on pourrait obtenir pendant neuf mois de l'année une puissance de $7.000 \mathrm{CV}$ environ.

5) Le bassin du Coghinas qui a une superficie de $2.477 \mathrm{~km}^{2}$ est le deuxième par importance dans l'Ile.

Des deux affluents principaux, le Rio Mannu di Ozieri et le Mannu di Berchidda, seul le deuxiéme s'écoule sur des terrains dont la constitution géologique et morphologique se prête à une réalisation convenable de réservoirs artificiels.

La nature granitique du terrain justifie la présence du grand lac artificiel, dont la capacité est de $242 \times 10^{6} . \mathrm{m}^{3}$ qui alimente le plus important centre de production d'énergie hydroélectrique de la Sardaigne, dont la concession autorisée s'élève à $19.866 \mathrm{CV}$. Dans les environs du grand réservoir déjà réalisé, on en prévoit plusieurs autres bien moins importants. A Monte Olia sur le Rio Pedrosu, au moyen d'un barrage dont la hauteur serait de $46 \mathrm{~m}$. on constituerait un lac de $12 \times 10^{6} \mathrm{~m}^{3}$. Sur le Rio di Oschiri au lieu dit Pedro Oe l'érection d'un barrage de $41 \mathrm{~m}$. de hauteur permettrait la formation d'un réservoir de $8,4 \times 10^{6} \mathrm{~m}^{3}$ Sur ce même cours d'eau et à Val Su Marinu on pourrait obtenir un autre réservoir de $4,6 \times 10^{6} \mathrm{~m}^{3}$. La réalisation de ces réservoirs contribuerait énormément à régulariser le fonctionnement du grand réservoir de Muzzone dont la capacité est insuffisante pour la régularisation complète des débits variables dans la région. La zone irrigable est limitée à 2.500 hectares sur l'étendue de Compo Coghinas.

6) Ce bassin s'étend en partie sur le versant Est de l'lle et de l'extrême Nord jusqu'à l'embouchure du Flumendosa. Sur le cours d'eau le Vignola dans la localité la Balestra on prévoit un réservoir d'une capacité de $12,6 \times 10^{6} \mathrm{~m}^{3}$, formé au moyen d'un barrage haut de $28 \mathrm{~m}$. Le débit que l'on pourrait ainsi obtenir suffirait à irriguer 1.200 hectares.

Sur le cours d'eau le Liscia on prévoit la formation de deux réservoirs l'un à Luvenu que l'on obtiendrait au moyen d'un barrage dont la hauteur atteindrait $27 \mathrm{~m}$. et la capacité $27 \times$ $10^{6} \mathrm{~m}^{3}$ et l'autre à $\mathrm{Li}$ Foci dont la capacité atteindrait $16,8 \times$ $10^{6} \mathrm{~m}^{3}$. Une faible partie du débit dont l'on disposerait ainsi serait suffisante pour l'irrigation de la plaine dans le voisinage immédiat. Dans le bassin de la rivière du Posada on a prévu trois réservoirs, l'un sur le Mannu di Pitti à Coa é Talixi de $15,63 \times 10^{6} \mathrm{~m}^{3}$ au moyen d'un barrage de $63 \mathrm{~m}$. de hauteur, l'autre sur le Posada à Dispensa Cecchini de $23,69 \times 10^{6} \mathrm{~m}^{3}$ obtenu au moyen d'un barrage haut de $39 \mathrm{~m}$. et enfin un troisième sur le Mannu di Bitti à Iscra Longa, dont la capacité serait de $21,39 \times 10^{6} \mathrm{~m}^{3}$. Ici aussi dans cette région l'étendue cultivable, qui n'est que de 2.500 hectares, n'absorberait qu'une partie du débit disponible; les réservoirs constitueraient ainsi en même temps une réserve éventuelle pour la production d'énergie.

7) Le Flumendosa qui donne le nom à ce bassin est le cours d'eau qui entre tous ceux de Sardaigne présente les plus importants débits unitaires, ce qui est dû, à l'imperméabilité absolue du sol et à sa configuration topographique. Son cours est torrentiel, puisque, encore à quatre $\mathrm{km}$. de la côte, il s'encaisse dans une gorge rapide et étroite. De ce fait il est très apte à former des réservoirs d'eau au moyen de barrages faciles à exécuter, ce qui permet d'exploiter rationnellement son débit. Mais, puisque il n'existe pas au voisinage immédiat des plaines qui bénéficieraient de ces réserves d'eau, on prévoit de les diriger sur le Campidano Nord di Cagliari, où il n'y a pas des quantités suftisantes d'eau pour revaloriser cette vaste plaine. 
Sur le haut Flumendosa on prévoit la construction de trois barrages destinés à la production d'énergie hydroélectrique : l'un sur le Sicea d'Erba à Bau de Muggeris qui aurait $57 \mathrm{~m}$. de hauteur et retiendrait $48 \times 10^{6} \mathrm{~m}^{3}$ d'eau ; l'autre à Bosco Corongiu sur le cours moyen du Flumendosa aurait $86 \mathrm{~m}$. de haut et serait capable de former un réservoir de $82,65 \times 10^{6} \mathrm{~m}^{3}$; enfin un troisième plus en aval à Is Stancas n'aurait que $11 \times 10^{6} \mathrm{~m}^{3}$ et permettrait de compléter l'utilisation des débits du Bosco Corongiu.

Bien plus importantes seraient les réserves qu'on pourrait obtenir au moyen de deux barrages à ériger l'un à Bruncu Sa Murra sur le Flumendosa, qui serait haut d'environ $92 \mathrm{~m}$. et l'autre sur le Flumineddu di Ussassai à Sa Brecca, qui dans l'ensemble formeraient une réserve de $388 \times 10^{6} \mathrm{~m}^{3}$ d'eau. L'utilisation rationnelle de cette eau nécessite la construction d'une galerie longue de $24 \mathrm{~km}$., qui pourrait s'effectuer en entreprenant deux "attaques" intermédiaires. Cette galerie permettrait de diriger l'eau de ces réservoirs dans le Campidano Nord di Cagliari pour y irriguer 5.700 hectares, après l'avoir utilisé pour la production de l'énergie. Il s'agit bien là d'installations grandioses, mais les résultats que l'on pourrait obtenir justifient une pareille entreprise. Toute cette exécution est susceptible d'être réalisée par étapes en harmonie avec le laps de temps qu'exige la transformation profonde et complète de la vie agraire, dont la réalisation est absolument nécessaire, ainsi qu'à la solution du problème démographique qui y est inséparablement lié.

Les derniers réservoirs prévus dans le bassin du Flumineddu seraient : un à Monté Scrocca avec une capacité de $11 \times 10^{6} \mathrm{~m}^{3}$ au moyen d'un barrage de $40 \mathrm{~m}$. de hauteur, cette réserve servirait à l'irrigation du territoire de Ballau et de Armungia ; l'autre à Naraghe Is Scrocca qui, au moyen d'un barrage de $33 \mathrm{~m}$. de hauteur, donnerait une réserve de $49 \times 10^{6} \mathrm{~m}^{3}$ utilisable pour la revaloriation de la zone du littoral du Sarrabus.

Sur le programme d'exécution des réservoirs du Flumendosa les avis sont partagés. Il n'est toutefois pas opportun de les envisager ou critiquer étant donné que cet exposé n'a pour but que d'éclairer, l'opinion sur les possibilités qui existent, mais dont le développement et l'usage sera uniquement défini par la suite et à son temps.

8) Ce demier bassin qui s'étend entre le bassin du Flumen. dosa et celui du Flumini Mannu est le moins important. Son noyau est constitué par le massif granitique dei Sette Fratelli qui s'avance au Sud jusqu'à la mer du Capo Carbonara. Le seul cours d'eau d'une certaine importance est le Sa Picocca sur lequel est prévu un réservoir à Monte Acuto qui aura une capacité de $34 \times 10^{6} \mathrm{~m}^{3}$, au moyen d'un barrage haut de $53 \mathrm{~m}$,

Enfin un autre réservoir artificiel est proposé : l'étang lo Stagno di Maracalagonis, dont le niveau serait rehaussé par la formation de deux modestes remblais de terre hauts de $9,50 \mathrm{~m}$. La capacité de ce réservoir serait de $19,30 \times 10^{6} \mathrm{~m}^{3}$ et puisque dans la Campidano oriental de Caglieri est très divulguée la culture de la vigne, qui ne demande pas d'eau, on peut done considérer cette réserve d'eau comme suffisante pour irriguer une étendue de 2.000 hectares.

\section{$* *$}

Comme il résulte de cet exposé les possibilités de construction de lacs artificiels en Sardaigne sont telles qu'elles suffiront entièrement pour une profonde transformation de l'économie agraire de l'lle.

Il ne s'agit pas ici de concessions nébuleuses, destinées à disparaitre au premier examen un peu plus sérieux, mais d'ouvrages imposants et toutefois d'exécution pratique très possible, car celle-ci a été étudiée sur place et les résultats ont été définit ivement établi à l'aide de données hydrologiques sûres. La réalisation de ces projets n'attend que le moment historique et économique propice. Celui ci semble, de l'avis d'éminents techniciens Italiens, assez proche. On signale en effet dès à présent des initiatives qui promettent la réalisation assez proche des installations du Flumendosa et du réservoir de MIonte Pranu sur le Rio di Palunas.

\section{G. Kolovitch.}

Ingénieur I. E. G. 